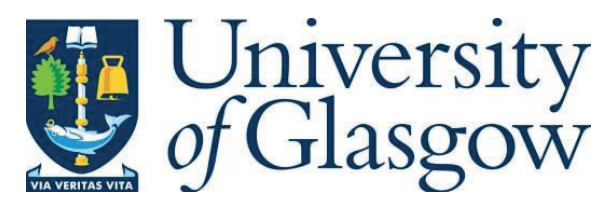

Monteiro, T.P., Ramesh, K., Silvestre, F. and Silva, R.G.A. (2020) Coupled framework for limit-cycle oscillations modeling based on leading-edge vortex shedding. Journal of Fluids and Structures, 99, 103137. (doi: 10.1016/j.jfluidstructs.2020.103137)

There may be differences between this version and the published version. You are advised to consult the publisher's version if you wish to cite from it.

http://eprints.gla.ac.uk/224467/

Deposited on: 2 November 2020

Enlighten - Research publications by members of the University of Glasgow http://eprints.gla.ac.uk 


\title{
Coupled Framework for Limit-Cycle Oscillations Modeling Based on Leading-Edge Vortex Shedding
}

\author{
Monteiro, T. P., Ramesh, K., Silvestre, F. \\ Instituto Tecnológico de Aeronautica, Brazil, University of Glasgow, UK, \\ Technische Universität Berlin, Germany
}

June 21, 2019

\begin{abstract}
Current trends in the aircraft industry involve higher aspect-ratio wings made of lighter materials. These trends seek to reduce fuel emissions and increase flight efficiency by reducing drag to lift ratio and overall weight, respectively, of the aircraft. This results in reduced structural stiffness and coupling between the aeroelastic modes and flight dynamics. The flutter phenomenon is of particular interest for aeroelastic studies, and modeling post-flutter limit-cycle oscillations (LCO) is a challenging problem.

Several studies have been developed to allow fast simulations of the highly non-linear aerodynamic situations, with leading-edge vortex modulation been a proved solution for modeling some forms of LCOs in airfoils. This article proposes a framework based on the 3D expansion of this method using strip theory and coupling with modal structural model for simulations of aerodynamic based nonlinear phenomenon. A cantilevered flat plate is used for testing and validating the framework against wind-tunnel experiments and the industry standard approach. The results show that the proposed model is able to capture the main behavior of the LCO observed in the experiments and is directly comparable with the current approaches used at the industry. The framework allows for scalability and is also fast enough to provide time-based results in under two days for a desktop simulation, reducing the need of expensive cluster computations. Finally, since it is completely physics-based it allows for the engineer to get insights on the aerodynamic flow at a fraction of the cost of more detailed CFD models.
\end{abstract}

KEYWORDS: aerodynamics; vortex-based methods; aeroelasticity; flutter; limit-cycle oscillations

\begin{tabular}{|ll|}
\hline LCO & Limit-Cycle Oscillation \\
\hline CFD & Computational Fluid Dynamics \\
\hline FEM & Finite Elements Method \\
\hline HALE & High-Altitude Long Endurance \\
\hline HPC & High Performance Computing \\
\hline PC & Personal Computer \\
\hline TEV & Trailing-edge Vortex \\
\hline LEV & Leading-edge Vortex \\
\hline LESP & Leading Edge Suction Parameter \\
\hline LDVM & LESP-Modulated Discrete-Vortex Method \\
\hline UVLM & Unsteady Vortex-Lattice Method \\
\hline
\end{tabular}

Table 1: Nomenclature

\section{Introduction}

The aerospace industry worldwide is seeking to develop "green aircraft" which are cleaner, quieter and more efficient, based on demands for reduction of fuel burn, emission of pollutants and costs of operation. This has motivated studies into new aircraft designs and configurations by leading aircraft manufacturers such as Airbus, Boeing and Embraer. Most of these futuristic designs involve a higher level of structural flexibility than in conventional aircraft. Even currently, the trends in the aviation industry are to increase wing span (to reduce induced drag) and maximize use of composites (to reduce weight), which lead to 
increased structural flexibility. Flexible aircraft structures result in the aeroelastic behavior becoming closer in frequency to that of the flight dynamics. Current procedures for aeroelastic, flight mechanics, and flight control law design, which are based on decoupling between flight dynamics (rigid-body) and aeroelastic dynamics, may not be valid for flexible aircraft [1].

Therefore, modeling flexible aircrafts introduces the challenge of coupling flight mechanics and aeroelasticity into the same framework. Murua et al. [2] and Hesse et al. [3] used a modal analysis structural formulation coupled with the Unsteady Vortex-Lattice Method to achieve medium-fidelity dynamic models that modeled aeroelasticity, with Silvestre et al. [4] validating the modal approach coupled with an aerodynamic indicial solution for slightly flexible aircraft, where the amplitude of structural deformation is expected to remain small. Palacios et al. [5] tested several aerodynamic models, and concluded that the indicial method provided good results for high-aspect ratio wings when compared to UVLM, as long as the amplitudes remained low. As a result, these methods provided good results for scenarios that presented linear behavior for aerodynamic and structure dynamics.

However, a common concern in aeroelasticity that has major effects on aircraft flight dynamics is flutter. This phenomenon occurs when the combined damping from aerodynamics and structural rigidity becomes negative, and a self induced, oscillatory motion begins on the airframe. Owing to nonlinearities typically present in the aerodynamics and structure, the oscillatory motion may have a finite and limited amplitude, and this is characterized as a Limit-Cycle Oscillation (LCO). These oscillations may cause structural failure and hence it is important to be able to predict their occurrence and model their effects in various flight regimes.

Flutter has been modeled with linear analysis, using linear aerodynamic theories such as those by Theodorsen [6, 7], in the frequency domain, and Wagner's indicial solution [8], in the time domain. As LCOs are by their very nature nonlinear, these linear models are not capable of predicting all possible LCOs and their features. This inability to fully predict LCOs creates the need for extensive flight testing, which is an expensive and time-consuming endeavor. These problems are present all the more in highly flexible aircraft like High Altitude Long Endurance (HALE) or futuristic configurations and designs with large-aspect-ratio wings. In these aircraft, the aeroelastic behavior may be strongly influenced by the flight dynamics. A nonlinear aeroelastic analysis tool capable of predicting all aspects of LCO behavior is hence desirable [9].

A combined formulation of aeroelasticity and flight dynamics, using Computational Fluid Dynamics (CFD) for aerodynamics and Finite Element Method (FEM) for structural dynamics is an expensive problem, requiring several hours of computational time even with High Performance Computing (HPC) resources. Semi-empirical methods such as the Beddoes-Leishman [10] or ONERA dynamic stall model [11] [12] are typically used for inexpensive aeroelastic analysis in such regimes. These are computationally fast but do not provide insight into the flow phenomena and are only valid in regimes where they have been previously validated. Recognizing the need for a new class of low-cost and physics-based methods, low-order unsteady flow solvers based on phenomenological augmentataion of unsteady potential-flow methods, such as those by Ramesh et al. [13] and Eldredge \& Wang [14] have been developed recently.

Structural non-linearities in the aeroelastic system can arise from large deformations, material properties, or loose linkages [15]. Lee et al. [15] have comprehensively reviewed the effects of structural nonlinearities on airfoil aeroelasticity, focusing on different types of nonlinear spring behavior such as bilinear or cubic variation in stiffness. In these studies, it is assumed that aerodynamics is linear, that is, the flow is incompressible, inviscid and attached to the airfoil. Aerodynamic nonlinearities may result from compressibility or viscous effects [15]. Tang et al. [16] studied flutter and LCOs of 2D panels through linear theory and experiment, and noted that linear theory is unsuccessful in predicting the large-amplitude LCOs that occur owing to aerodynamic nonlinearities. Nonlinear aerodynamics resulting from viscous flow phenomena is largely dependent on the Reynolds number and the reduced frequencies involved, and leading-edge vortices (LEVs) have been seen to play a crucial role. In helicopter and wind-turbine applications that are characterised by large Reynolds numbers and low reduced frequencies, LEVs and the resulting dynamic stall phenomenon might lead to violent vibrations and mechanical failure [17]. Conversely, LEVs in high-frequency flows at low Reynolds numbers are responsible for the success of high-lift flight in insects [18, 19, 20, 21], and high propulsive [22] and power-extraction [23] efficiencies.

The type of nonlinearity in the system may result in either subcritical or supercritical LCOs, which occur below and above the flutter velocity, respectively. For example, the structural nonlinearity of soft cubic stiffening results in subcritical LCOs that depend on initial conditions, while hard cubic stiffening results in supercritical LCOs that are independent of initial conditions. With regard to aerodynamic nonlinearities, Dowell and Tang [24] have reported that trailing-edge flow separation results in subcritical LCOs that depend on initial conditions. On the other hand, Ramesh et al. [25] have shown that 2D LEVs 
cause supercritical LCOs that are independent of initial conditions.

In this research, we are specifically concerned with aerodynamic non-linearities and limit-cycle oscillations of a constrained high-aspect-ratio wing resulting from leading-edge vortex shedding at low Reynolds numbers.

Cantilevered wings have been widely used in the development and validation of LCO behavior. Tang and Dowel [26] modeled LCO behavior of a cantilevered wing using a non-linear structural model coupled with a linear vortex-lattice aerodynamic model. Preidikman and Mook [27], on the other hand, used a linear structural model coupled with a non-linear Unsteady Vortex-Lattice Method to model LCO on cantilevered wing. Finally, Patil et al. [28] modeled a complete HALE aircraft with non-linear structural and aerodynamic models and concluded that cantilevered wing model could give good aeroelastic results when considering a trimmed HALE aircraft.

Therefore, an experiment based on a cantilevered flat plate with a tip ballast $[26,29,30]$ is designed in such a manner that LEVs are the dominant non-linearity in the system.

Ramesh et al. [31] have developed time-stepping unsteady airfoil theory based on potential flow, which holds valid uniformly regardless of amplitude and reduced frequency of motion, and shape of trailing wake. This method was seen to predict aerodynamic forces well even under challenging conditions of large amplitude and high reduced frequency. To model flows with leading edge vortices(LEVs) [46], the Leading Edge Suction Parameter(LESP) was developed by Ramesh et al [32] to predict the onset of LEV formation. This parameter is a measure of the suction at the leading edge and it was shown that initiation of LEV formation always occurred at the same critical value of LESP, regardless of motion kinematics so long as the airfoil and Reynolds number of operation were the same. Using this criterion, a $2 \mathrm{D}$ discretevortex method was developed in which the LESP criterion was used to modulate the initiation, growth and termination of leading-edge vortices [13]. This method is abbreviated as LDVM (LESP-Modulated Discrete-Vortex Method), and has been successfully applied to study post-flutter limit-cycle oscillations of an airfoil (2D) constrained by torsional and translational springs [25].

Ramesh et al. [33] have applied the LDVM formulation to finite-wing (3D) flows using strip theory and a correction based on lifting-line theory. Hirato [34] and Monteiro et al. [35] have attempted to adapt the LESP concept to Unsteady Vortex-Lattice Methods (UVLMs) for finite wings. In this research, a simple strip theory is used for the aerodynamic modeling, with the LDVM formulation implemented along each strip. This is coupled with a linear structural model that is based on elastic modal shapes. The ability of this model to capture post-flutter LCOs caused by LEV shedding is studied by comparing the simulation results against experimental data.

\section{Numerical methods for 3D aeroelastic model}

\subsection{Nonlinear aerodynamic model (LDVM)}

The LDVM aerodynamic model which is based on the 2D discrete-vortex method is used as the basis for the numerical modeling employed in this research. The model is extended to 3D using strip theory. This approximation is justified for the high-aspect-ratio wing considered in this research. The 2D LDVM method is first summarized below. The interested reader may refer to Refs. [13, 31] for further details.

\subsubsection{Large-angle unsteady thin-airfoil theory}

At the foundation of the LDVM is a large-angle unsteady thin-airfoil theory detailed in Ramesh et al. [31]. This theory is based on the time-stepping formulation given by Katz \& Plotkin [36], but eliminates the traditional small-angle assumptions in thin-airfoil theory which may be invalid in flows of current interest. At each time step, a discrete vortex is shed from the airfoil trailing edge (referred to as TEV) as shown in figure 1. When dictated by the LESP-based shedding criterion (section 2.1.2), a discrete vortex is also shed from the leading edge at some time steps. The vorticity distribution over the airfoil at any given time step is taken to be a Fourier series truncated to $r$ terms:

$$
\gamma(\theta)=2 U\left[A_{0} \frac{1+\cos \theta}{\sin \theta}+\sum_{i=1}^{r} A_{i} \sin (i \theta)\right]
$$

where the transformation variable $\theta$ relates to the chordwise coordinate as: $x=c(1-\cos \theta) / 2$, with $x$ measured from the leading edge; that is, $0 \leq x \leq c$ and $0 \leq \theta \leq \pi . A_{0}, A_{1}, \ldots, A_{r}$ are the time-dependent 


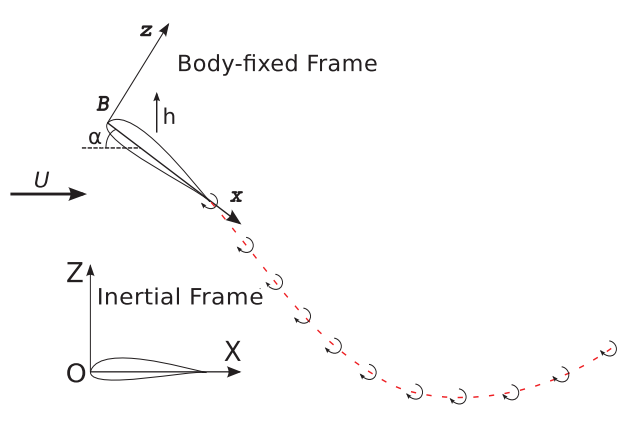

Figure 1: Depiction of time-stepping scheme.

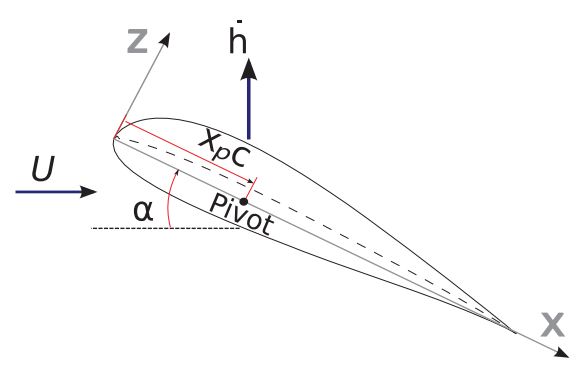

Figure 2: Airfoil velocities and pivot location.

Fourier coefficients, and $U$ is the freestream velocity. The Kutta condition (zero vorticity at the trailingedge) is enforced implicitly through the form of the Fourier series. The Fourier coefficients are calculated by enforcing the boundary condition of zero normal flow through the airfoil camberline as

$$
\begin{aligned}
& A_{0}=-\frac{1}{\pi} \int_{0}^{\pi} \frac{W(\theta)}{U} d \theta \\
& A_{i}=\frac{2}{\pi} \int_{0}^{\pi} \frac{W(\theta)}{U} \cos (i \theta) d \theta
\end{aligned}
$$

where $W(\theta)$ is the induced velocity normal to the airfoil camberline. This value is calculated from components of motion kinematics, depicted in figure 2, and induced velocities from all vortices in the flowfield.

When there is no LEV shedding in a time step, the only unknown is the strength of the last-shed trailing-edge vortex and this is calculated iteratively such that Kelvin's circulation condition is satisfied [31].

\subsubsection{LESP criterion for LEV formation and shedding}

The LESP is a measure of the suction peak at the leading edge, which in turn is caused by the stagnation point moving away from the leading edge when the airfoil is at an angle of attack. From Garrick [37] and von Kármán \& Burgers [38], the suction at the leading edge in potential flow may be expressed as

$$
S=\lim _{x \rightarrow L E} \frac{1}{2} \gamma(x) \sqrt{x}
$$

Evaluating using the current formulation, $S=\sqrt{c} U A_{0}$. The Leading Edge Suction Parameter is defined as a nondimensional value of suction at the leading edge, and is hence simply set equal to the first coefficient from Eq. (1), $A_{0}$.

Katz [39] has noted that real airfoils have rounded leading edges which can support some suction even when the stagnation point is away from the airfoil leading edge. The amount of suction that can be supported depends on the airfoil shape and Reynolds number of operation. When these quantities are kept constant, it was shown in Ramesh et al. [13] that initiation of LEV formation always occurred at the same value of LESP regardless of motion kinematics and history. This threshold value of LESP, which is a function of the airfoil shape and Reynolds number, is termed the critical LESP. This value, for any given airfoil and Reynolds number (and other specific operating conditions such as freestream turbulence and the presence of roughness), can be obtained from CFD or experimental predictions for a single motion [13], and can then be used for any other motion to predict LEV formation. In the LDVM model, a discrete vortex is shed is from the leading edge at those time steps when the instantaneous LESP ( $A_{0}$ value) is greater than the critical LESP value. The strength of the LEV is determined such that the instantaneous LESP value, which would have otherwise exceeded the critical LESP value, is made equal to the latter. This condition, along with Kelvin's condition, is used to determine shed vortex strengths iteratively in time steps where both TEV and LEV are shed. 


\subsubsection{Vortex method details and Implementation}

In the current approach, the vortex-core model proposed by Vatistas et al. [40], which approximates the Lamb-Oseen vortex is used to model the discrete vortices as vortex blobs. In this model, the velocities induced at $X$ and $Z(u$ and $w)$ by the $k^{t h}$ vortex in the $X$ and $Z$ direction are:

$$
[u, w]=\frac{\gamma_{k}}{2 \pi} \frac{\left[\left(Z-Z_{k}\right),\left(X_{k}-X\right)\right]}{\sqrt{\left[\left(X-X_{k}\right)^{2}+\left(Z-Z_{k}\right)^{2}\right]^{2}+v_{\text {core }}^{4}}} .
$$

where $X_{k}$ and $Z_{k}$ are the positions of the $k^{t h}$ vortex. The vortex core radius, $v_{\text {core }}$, in the simulations was set as $0.02 c$, where $c$ is the section chord. To control vortex count and limit the computational cost, vortices that are a distance greater than ten chord lengths from the airfoil are deleted. A fixed nondimensional time step $\left(d t^{*}\right)$ of 0.015 was used to advance the solution in time. Further details about the choice of these parameters may be found in Ramesh et al. [13] [25]. The dimensional time step is calculated as $d t=d t^{*} \frac{c}{U}$.

The model was implemented in MATLAB, and the wake rollup routine was implemented as vectorized code since nested for-loops are inefficient in MATLAB. The strip theory approach, by design, separates the three-dimensional model into discrete, and independent, two-dimensional solutions. This makes it a perfect candidate for parallelization. The parallel implementation achieved varying performance boosts depending on the number of CPU workers made available. As a reference, the run time with a parallel pool of 3 workers on a Core i7 7700K for each time-step of physical simulation ranged from 0.18s (initial linear results) to $1.6 \mathrm{~s}$ (fully developed LCO) with 20 spanwise strips generating LEV.

In recent work, SureshBabu et al. [41] and Darkananda et al. [42] have developed model-reduction methods for discrete-vortex methods where amalgamation of vortex blobs is implemented to drastically reduce the vortex count and speed up the code. In the current research, such model reduction methods were not used.

\subsubsection{Force and Moment Calculation}

Once the vorticity distribution on the airfoil is determined, the normal $\left(c_{n}\right)$ and suction $\left(c_{s}\right)$ coefficients at a wing strip are calculated by equations 6 and 7 respectively.

$$
\begin{aligned}
& c_{n}=\frac{F n}{q_{\infty} c}=2 \pi\left[\frac{(U \cos \alpha+\dot{h} \sin \alpha)}{U}\left(A_{0}+\frac{1}{2} A_{1}\right)+\right. \\
&+\frac{3 c}{4 U} \dot{A}_{0}+\frac{1 c}{4 U} \dot{A}_{1}+\frac{1 c}{8 U} \dot{A}_{2}+\frac{2}{U^{2} c} \sum_{n=1}^{n d i v}\left(\frac{\partial \phi_{\text {lev }}}{\partial x}+\frac{\partial \phi_{\text {tev }_{n}}}{\partial x}\right) \gamma(x, t) \\
& c_{s}=\frac{F_{s}}{q_{\infty} c}=2 \pi A_{0}^{2}
\end{aligned}
$$

Where, $\phi_{l e v_{n}}$ and $\phi_{t e v_{n}}$ are the velocity potential from leading edge vortices and trailing edge vortices respectively.

The lift coefficient is calculated by combining Normal and Suction coefficients (equation 8) and the moment coefficient, $c_{m}$ is calculated by dividing the moment around the pivot $\left(m_{e a}\right.$ given by equation 9 , where $x_{e a}=X_{p} c$ and $X_{p}$ is the pivot position as a fraction of the chord) by $\frac{1}{2} \rho U^{2} c^{2}$.

$$
\begin{gathered}
c_{l}=c_{n} \cos (\alpha)+c s \sin (\alpha) \\
m_{e a}=x_{e a} F_{N}-\rho \pi c^{2} U\left[(U \cos (\alpha)+\dot{h} \sin (\alpha))\left(\frac{1}{4} A_{0}+\frac{1}{4} A_{1}-\frac{1}{8} A_{2}\right)+\right. \\
\left.+c\left(\frac{7}{16} \dot{A}_{0}+\frac{11}{64} \dot{A}_{1}+\frac{1}{16} \dot{A}_{2}-\frac{1}{64} \dot{A}_{3}\right)\right]-\rho \sum_{n=1}^{n d i v}\left(\frac{\partial \phi_{l e v_{n}}}{\partial x}+\frac{\partial \phi_{\text {tev }}}{\partial x}\right) \gamma(x, t) x
\end{gathered}
$$

These are applied at each strip center and integrated over the span to calculate the wing total lift and moment. Details of this operation are given in section 2.2.3. 


\subsection{Structural model}

The main focus of this work is to study the influence of aerodynamic nonlinearities on post-flutter LCOs, and the ability of the strip-theory expanded LDVM in predicting such LCOs. Therefore, in order to achieve fast simulations, a linear structural model was defined based on a cantilevered flat plate, fixed vertically, and with a ballast positioned at the wing tip with its center not coincident with the elastic axis of the wing. The center of the ballast was moved towards the leading-edge, away from the elastic axis. This preset offset reduces the flutter velocity of the system and generates coupled bending-torsion modes. The flat plate was modeled by a finite elements model, which is the base for defining the plate's modes of flexibility. This model also allows some insight on the expected flutter behavior of this system, and the modal structural model built from the modes provides a fast, linear, structural model that is often used in flexible aircraft simulation.

\subsubsection{Modes of Flexibility}

To build the modal structural model, it is imperative to understand the system modes of flexibility and define the modes of flexibility that are activated by the flutter phenomenon. Figure 3 shows the first four modes of flexibility for the cantilevered flat plate with an offset ballast at the tip. These modes are obtained from a Finite Elements Structural Model. This model uses 70 beam elements with six degrees-of-freedom each. The Finite Elements model is based on the formulation detailed on [43].
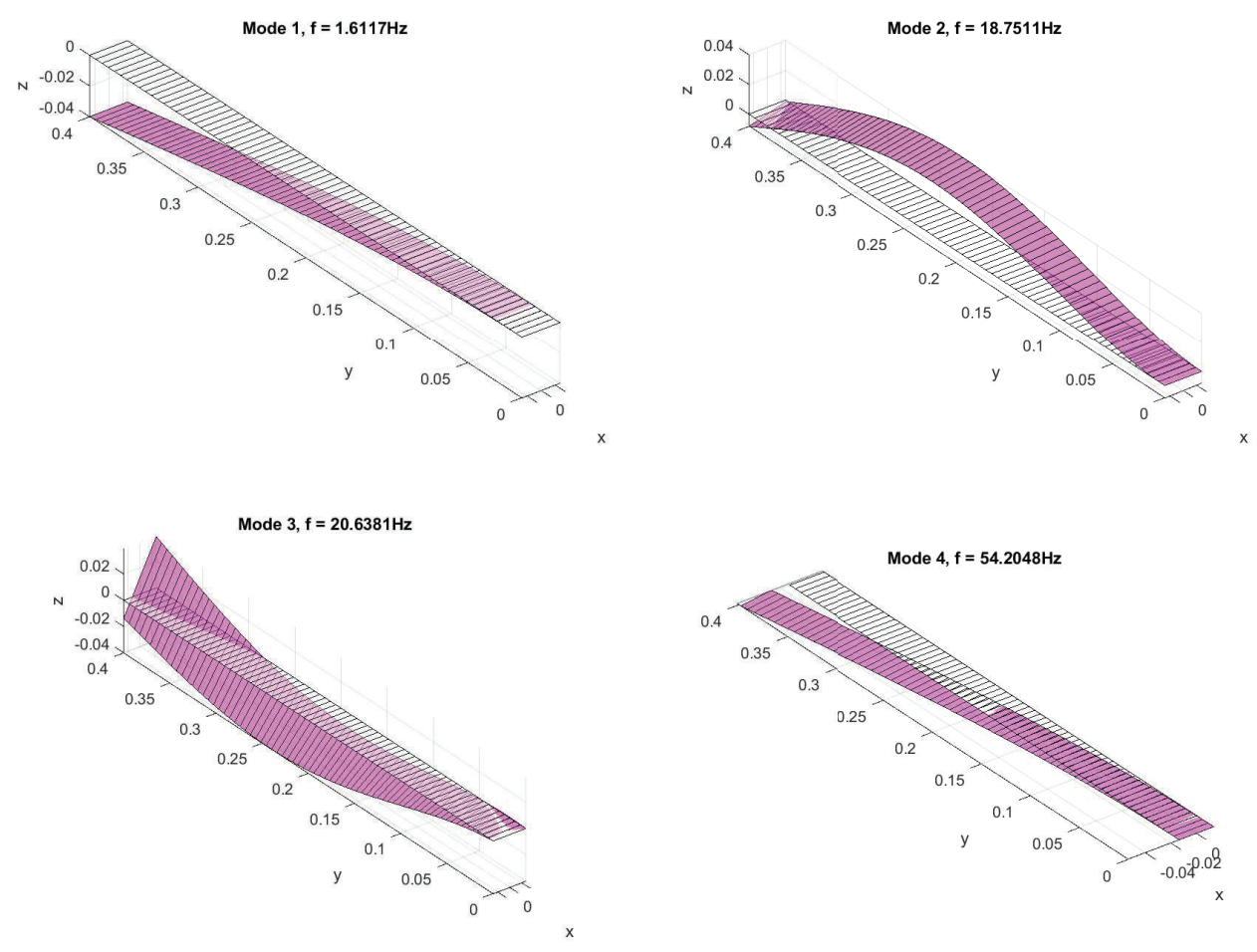

Figure 3: Flat plate with ballast modes of flexibility

The first mode, shown on the top left of Fig. 3, is a pure bending mode, that will affect all the strips, especially those strips near the wing tip. It operates at a lower frequency and, in flutter situations, is usually not excited enough to influence the resulting LCO's amplitude. For this case, however, it was possible to see from the resulting LCO a clear presence of the first bending mode.

The second and third modes are the main modes responsible for flutter oscillations for this aeroelastic system. Since they operate at close frequencies, they tend to coalesce and oscillate at a fixed amplitude LCO. The second mode, top right graphic, is the second bending mode, that presents some torsion due to the offset in the ballast. The third mode, bottom left graphic, is a mostly torsion mode. Like the second mode, the offset ballast produces some coupling with bending movements. 
Finally the fourth mode is responsible for bending in the direction of the freestream, and it operates at a much higher frequency and has a more stiff response. With flutter behavior being mostly due to the second and third modes, as the model simulation and experimental results below show. Due to its increased inertia in the chordwise axis it shouldn't be excited in the simulation, however, since the next vibration mode has a frequency of around $59.7 \mathrm{~Hz}$ no extra modes will be included in this simulation, as experimental results are filtered above $55 \mathrm{~Hz}$ due to electrical interference from the grid.

\subsubsection{Variable Transformation}

The structural model is based on the above defined modes of flexibility. The model is defined starting from the dynamic equation of motion (Equation 10).

$$
M \ddot{q}+C \dot{q}+K q=F
$$

where $\mathrm{M}$ is mass matrix, $\mathrm{C}$ is the damping matrix, $\mathrm{K}$ is the stiffness matrix and $\mathrm{F}$ are the forces and moments. By writing the physical coordinates as equation 11, it is possible to express the deformations as a combinations of modal shapes $(\eta)$. These can be truncated at the previously defined modes to allow for order reduction and fast simulations.

$$
q=\Lambda \eta
$$

where $\Lambda$ is the eigenvector matrix representing the modal shapes and $\eta$ is the generalized displacements. The equations of motion can be rewritten as:

$$
\ddot{\eta}_{m \times 1}+2 \epsilon_{m \times m} \Omega_{n_{m \times m}} \dot{\eta}_{m \times 1}+\Omega_{n_{m \times m}}^{2} \eta_{m \times 1}=\mu_{m \times m}^{-1} Q^{f}{ }_{m \times 1}
$$

Where,

$$
\begin{array}{r}
\mu=M \Lambda \\
\Omega^{2}=K \Lambda \\
2 \epsilon \Omega=C \Lambda
\end{array}
$$

with $m$ being the number of modes used at the truncation, $\epsilon$ being the diagonal matrix of modal damping (equation 13c), $\omega_{n}$ the diagonal matrix of modal natural frequencies (equation 13b), $\mu$ the diagonal matrix of modal masses (equation 13a) and $Q^{f}$ the vector of generalized forces [4] [44].

\subsubsection{Coupling between the aerodynamic and structural models}

The coupled model is, therefore, defined by modeling the $Q^{f}$ vector of generalized forces.

The structural model defined in this article was discretized in 20 strips. For each strip, the structural model feeds the strip's kinematics (equations 14 and 15) to the 2D LDVM model described below. In these equations $\Lambda^{n_{s}}$ is the eigenvector matrix evaluated at strip's $n_{s}$ location. The force and moment coefficients are then calculated and applied to the structure.

$$
\begin{aligned}
& {\left[\begin{array}{l}
\alpha^{n_{s}} \\
h^{n_{s}}
\end{array}\right]=\Lambda^{n_{s}} \eta} \\
& {\left[\begin{array}{l}
\dot{\alpha}^{n_{s}} \\
\dot{h}^{n_{s}}
\end{array}\right]=\Lambda^{n_{s}} \dot{\eta}}
\end{aligned}
$$

The generalized forces $Q^{f}$ can be written as a function of the lift coefficient $\left(c_{l}^{n_{s}}\right)$ and moment coefficient $\left(c_{m}^{n_{s}}\right)$ given by the aerodynamic model at each strip, and integrating them for the full wing. Equation 16 shows generalized forces calculation.

$$
Q^{f}=\sum_{n_{s}=1}^{n_{\text {strip }}} \Lambda q_{\infty}\left[\begin{array}{l}
-c_{l}^{n_{s}} c \Delta y \\
c_{m}^{n_{s}} c^{2} \Delta y
\end{array}\right]
$$

Where $q_{\infty}$ is the dynamic pressure, $c$ is the strip chord, $\Delta y$ is the strip length and $n_{s}$ is the strip number.

Vortex-based methods are usually more suited for calculating lift and moment coefficients, with pressure drag caused by LEVs being typically overestimated. Since from the modes of flexibility it is expected 
that drag force (direct responsible for the fourth mode) will not play an important role, it is not applied to the current model.

The chart below (figure 4) summarizes the coupled model execution process. A fourth-order RungeKutta fixed-step solver was used to solve the coupled model dynamics, with the aerodynamics being executed once per time step. The fundamental time-step used was set by the aerodynamic model following the studies of Ramesh et al. [25].

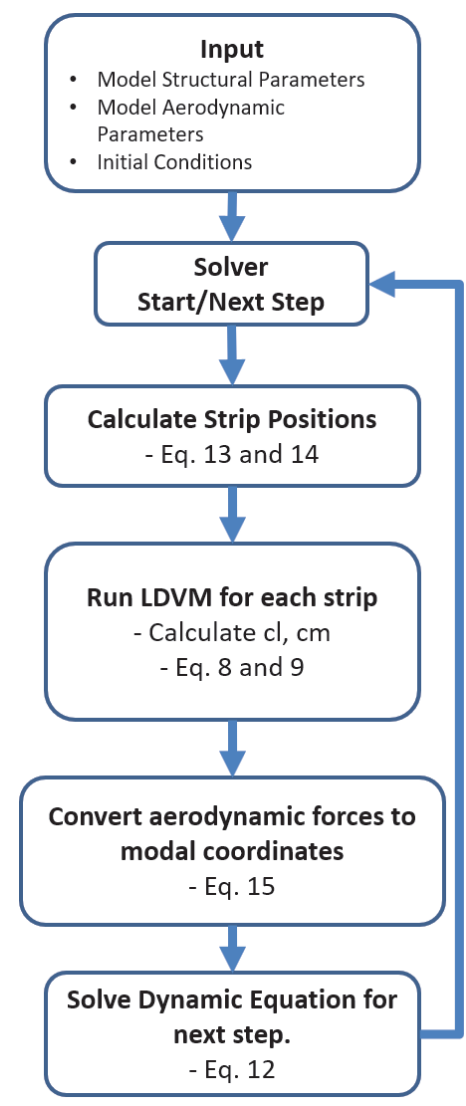

Figure 4: Coupled model execution chart

\subsubsection{Limitations of the Model}

The current implementation does not take into account any $3 \mathrm{D}$ effects for the aerodynamics. This means that the forces generated by the strips at the wing tip are not affected by the tip vortices and are effectively overestimated for the quasi-steady case. However, this simplification is acceptable, because for the unsteady case studied in this article the effect can be harder to predict since the higher movement at the wing tip generates a strong downwash that can have a higher effect than the tip vortices. Moreover,. for high-aspect ratio wings, such as the one in this article, this simplification should have only minor effects in overall forces calculations.

As stated in previous works with LDVM, it does not take into account trailing edge separation, making the model ill suited to simulate situations where trailing edge separation has major effect on the overall dynamics. However, as the current study has been performed with a flat plate, LEV shedding is expected to be dominant effect on the aerodynamics. Although capable of calculating the feasible forces at high angles of attack, a 90 degree angle of attack limit is imposed to the model due to the continuous characteristic of the sine and cosine force composition. For instance, a positive 100 degree angle of attack would be equivalent to a negative 80 degrees physically, but the model would not correctly reproduce this condition. Also, even though the wing's pivot position varies along the chord in the structural model, due to the offset in the ballast, the LDVM implementation approximates the pivot position constant at the middle of chord for the entire wing. This simplification should have only minor effects in the results, since the offset used is small. 
Finally, the structural model is strictly linear and approximates deformations by linearly combining the modal shapes. Therefore, structural non-linearities of any form can not be simulated by this model.

\subsubsection{Computational Cost}

One of the main benefits of the proposed framework is the low computational cost, when compared to the standard CFD/FEM models used for fluid-structure interaction with nonlinear aerodynamics.

The current model can be executed in a standard desktop computer, with tests executed in this article taking an average of $0.69 \mathrm{~s}$ for each step running on a Core i7 CPU 7700k at the base clock speeds. This depends greatly on the distribution of leading-edge vortices on the wake, with steps that do not have any LEV taking an average of 0.18 s per simulation step, and steps with LEV shedding and rollup in all strips taking an average of $1.6 \mathrm{~s}$ per simulation step.

The model is also scalable by the nature of the strip theory approach, that allows for parallel execution of the aerodynamics of the $2 \mathrm{D}$ strips. The discretization of the aerodynamics in 20 strips also took into into account the diminished return in accuracy when more strips were used versus the cost of running the extra 2D models.

\subsection{Linear aerodynamic model}

As an initial verification procedure, the LDVM-based model was verified against industry standard, potential-theory-based 2D implementation for time-based flutter analysis. This model consists of a modalbased structural model (the same used in the coupled model proposed), with a Wagner aerodynamic solution approximated by the Jones' exponential function.

The aerodynamic force and moment for this approximation are calculated by:

$$
l=l_{N C}+2 \pi \rho U(c / 2) Q \phi(s)
$$

and,

$$
m_{e a}=m_{e a(N C)}-\pi \rho U(c / 2)^{2} Q+2 \pi \rho U(c / 2)^{2} Q \phi(s)(-(a+1 / 2))
$$

where $l_{N C}$ and $m_{e a(N C)}$ are the non-circulatory portions of the lift force and moment around the elastic axis, $a$ is the pivot position from the half-chord position $\left(a=2 X_{p}-1\right), Q$ is the kinematic influence given by equation 19 and $\phi(s)$ is the Wagner function, which can be approximated by the Jone's exponential expression (equation 20) [4] [45].

$$
\begin{gathered}
Q=U \alpha+\dot{h}+(c / 2)(1 / 2-a) \dot{\alpha} \\
\phi(s)=1-0.165 e^{-0.041 s}-0.335 e^{-0.32 s}
\end{gathered}
$$

\subsection{Model Parameters}

The model developed was tested using a cantilevered aluminum beam. Apart from being a very well documented case, it is well suited for experimental validation, allowing for simplified testbench setup. The wing parameters, shown on table 2 were based on a slender plate with the following characteristics. The ballast inertia about the YY axis (spanwise axis) has been neglected, since it is much smaller than $\mathrm{ZZ}$ and XX axis (normal force and chordwise axis respectively). The structural model uses these values for the simulations.

For the aerodynamic model, LDVM was modeled using the standard values proposed by Ramesh et al. [25] for the 2D implementation, due to the similarities between the aerodynamic flow. Table below shows the values used for simulations. The Critical LESP values used in the simulations are defined in the graphics without the decimal point. The critical LESP value of 0.10 is considered a baseline for flat plates achieving an LCO, tests were conducted for other critical LESP values in order to verify possible differences in 2D and 3D LDVM models. The flat plate was simulated with a fixed 1 degree angle of attack. From the experiments the freestream velocity of $10 \mathrm{~m} / \mathrm{s}$ was defined as the flutter speed. 


\begin{tabular}{|ll|}
\hline Span $(m)$ & $400 \mathrm{e}-3$ \\
\hline Chord $(m)$ & $27 \mathrm{e}-3$ \\
\hline Thickness $(m)$ & $0.8 \mathrm{e}-3$ \\
\hline Elastic Coefficient $(P a)$ & $73.1 \mathrm{e} 9$ \\
\hline Wing Density $\left(\mathrm{kg} / \mathrm{m}^{3}\right)$ & 2780 \\
\hline Ballast Offset Position $(\mathrm{m})$ & $5 \mathrm{e}-3$ \\
\hline Ballast Mass $(\mathrm{kg})$ & 0.0328 \\
\hline Ballast Inertia $\left(I_{Z Z}\right.$ and $\left.I_{X X}\right)\left(\mathrm{kg} \cdot \mathrm{m}^{2}\right)$ & $1.858 \mathrm{e}-5$ \\
\hline
\end{tabular}

Table 2: Structural Model Parameters

\begin{tabular}{|ll|}
\hline Chord $(\mathrm{m})$ & 0.0270 \\
\hline Freestream velocity $(\mathrm{m} / \mathrm{s})$ & 10 \\
\hline Air density* $\left(\mathrm{kg} / \mathrm{m}^{3}\right)$ & 1.1 \\
\hline Number of strips & 20 \\
\hline Strip spanwise length $(\mathrm{m})$ & 0.02 \\
\hline Non-dimensional core radius of point vortices & 0.02 \\
\hline Number of divisions along chord on airfoil & 70 \\
\hline Number of Fourier terms used to compute vorticity at a location on chord & 46 \\
\hline Number of chords to start deleting vortexes & 10 \\
\hline Pivot position as a fraction of the chord $(0-1)$ & 0.5 \\
\hline Reference Reynolds number & 10000 \\
\hline Critical LESP & $0.10,0.05,0.03$ and 0.01 \\
\hline Non-Dimensional time step & 0.015 \\
\hline
\end{tabular}

Table 3: Aerodynamic Model Parameters (*Measured in experiment's room barometer)

\section{Experimental method}

Experiments were developed to validate the model and help define the values of critical LESP values for this situation. This differs from previous studies with LESP-based modulation, since they used computational fluid dynamics (CFD) for validating and tuning the LESP value. Since the LCO behavior presented by this experiment is mainly due to nonlinearities in the aerodynamic forces, it was possible to adjust the LESP critical value with these results without significant differences from what could be achieved from CFD methods. The experiments were conducted to represent a scenario of leading-edge generated flutter. The experiment setup is shown in figure 5 , and was performed by increasing the wind tunnel dynamic pressure (velocity) in steps of $0.5 \mathrm{mmH} 2 \mathrm{O}$ until flutter induced vibration was observed. After vibration was perceived the system was kept in a steady state until the LCO was reached.

A thin aluminum flat plate, with slightly rounded leading-edge, is positioned just outside the test section with a fixed angle of attack of 1 degree. The flat plate has a ballast added to the wing tip to reduce the flutter velocity and make it compatible with the working range of freestream produced by the wind tunnel. The ballast is positioned with $5 \mathrm{~mm}$ offset in the direction of the leading edge of the flat plate; this increases flutter response by coupling the bending and torsional vibration modes. Figure 6 shows a schematic of the wing with the ballast positioned and figure 7 shows it mounted in front of the wind tunnel.

The main sensors in this test apparatus were a laser vibrometer to measure the trailing edge velocity around $3 \mathrm{~cm}$ from the wing tip and a aerodynamic balance to measure the force produced at the wing root, that, although not used in this work, ended up influencing the experiment's LCO frequency. The studies resulted in a perceived LCO being achieve with the freestream velocity around $10 \mathrm{~m} / \mathrm{s}(6 \mathrm{mmH} 2 \mathrm{O}$ on the Betz Manometer). More information on the experiments setup and execution can be found on previous works [33] [35].

\subsection{Experiment Limitations}

The velocity of the freestream was controlled manually by verifying the Betz Manometer attached to the wind tunnel, however, due to the open-section nature of the tunnel, small variations were observed, with the mean velocity kept at $10 \mathrm{~m} / \mathrm{s}$. The tunnel presents a turbulence level of $0.5 \%$ that can affect the experiment's frequency response peak position and energy. Also due to the open-section nature of 


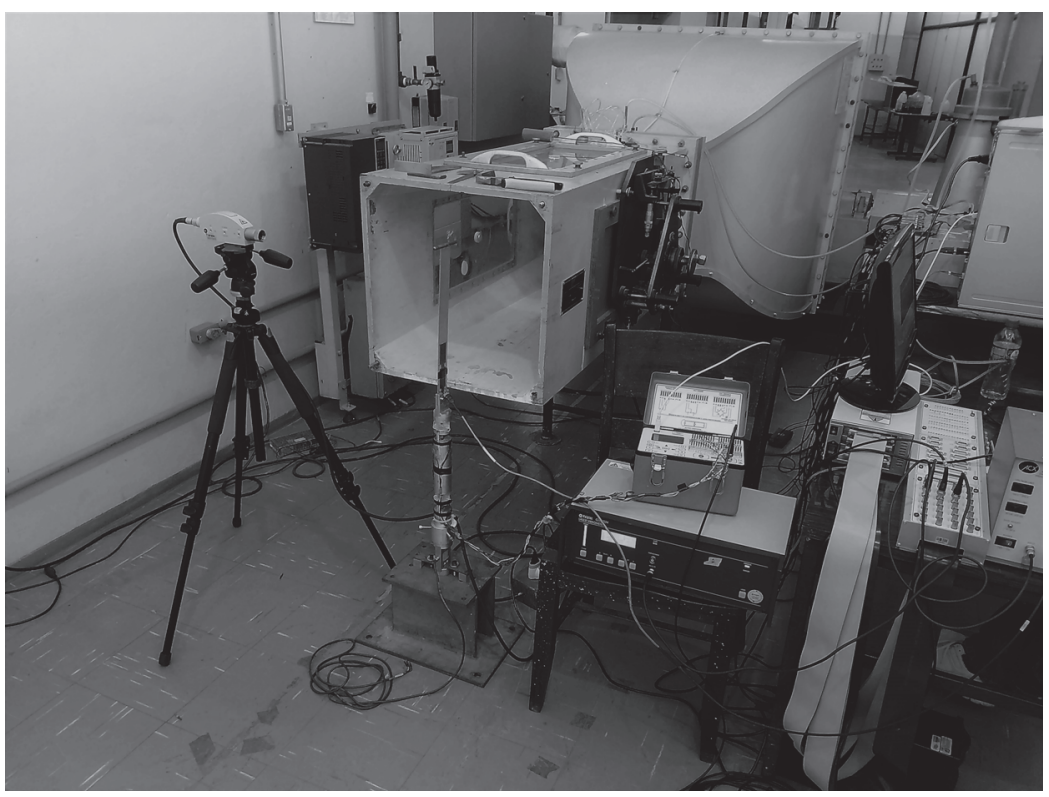

Figure 5: Experiment Setup

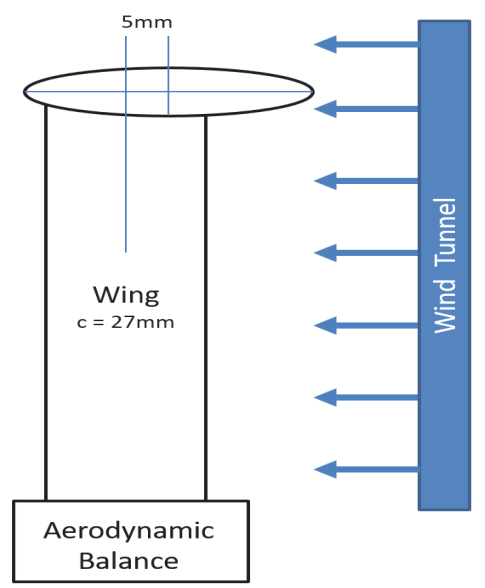

Figure 6: Flat plate position schematic

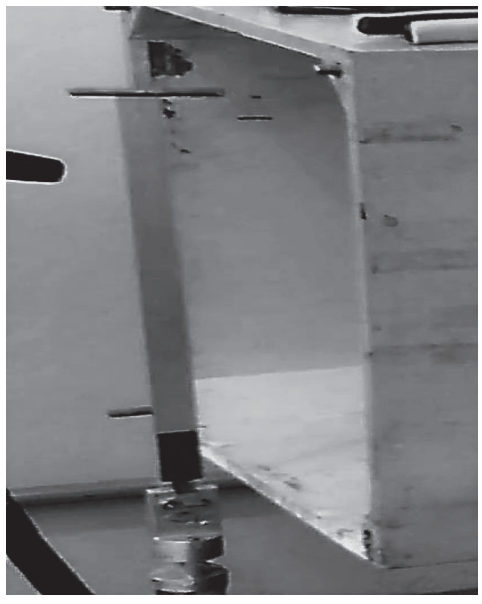

Figure 7: Flat plate position picture 
the tunnel, some lift could be generated by the aerodynamic balance's geometry, this is considered small enough due to the cylindrical shape of the balance and is therefore not relevant for the results measured. Finally, frequencies above $55 \mathrm{~Hz}$ are filtered out due to electrical interference from the grid.

However, due to the self excitement nature of the flutter phenomenon, a small vibration was perceived at the aerodynamic balance. This should have some minor effects on the amplitude of measured velocities of the laser vibrometer, affecting mainly the excited frequencies of the experiment. The full aeroelastic model only represents the wing's structural dynamics and the effects of the balance weren't modeled. This should allow for an amplitude focused comparison, with a qualitative verification of the frequencies. In order to assess the influence of the aerodynamic balance in the experimental results, a Finite-Elements model of the full apparatus, comprised of the balance, wing and ballast, was developed. The first six vibration modes were analyzed. Figure 8 shows the first five vibration modes, looking at the y axis, it is possible to see the balance plotted from 0 to $0.5 \mathrm{~m}$, and the wing plotted between $0.5 \mathrm{~m}$ and $0.9 \mathrm{~m}$. Since the sixth mode presents the frequency of $41.82 \mathrm{~Hz}$ it shouldn't have any effects in the results and was omitted from the figure. By verifying the modes auto-vectors it is possible to correlate modes 1,4 and 5 of the full apparatus with the wing modes 1,2 and 3 that are prominent in the flutter results. The full model, therefore, presents two new vibration modes: Mode 2, which is in the chordwise direction, does not influence the results, since the forces in this direction shouldn't be strong enough to generate any movement. Mode 3, however, should have a major impact in the results and can effectively lower the resulting $\mathrm{LCO}$ frequency.

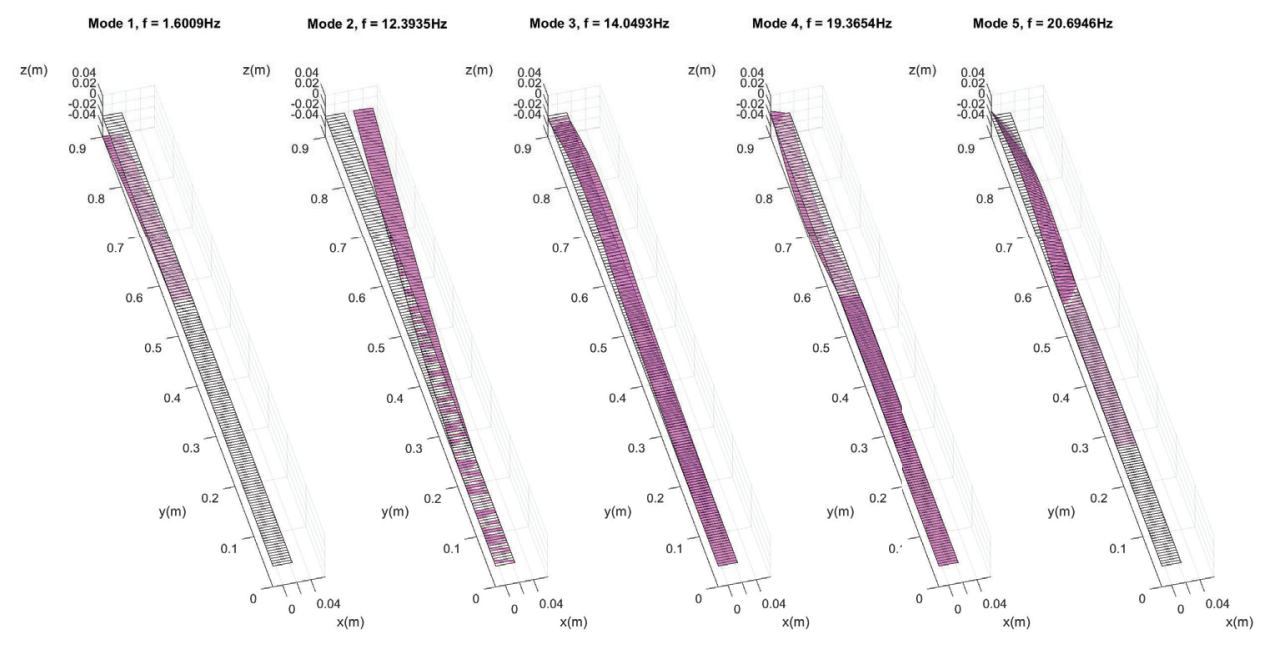

Figure 8: Full apparatus vibration modes

\section{Results and discussion}

\subsection{Comparison with Linear Aeroelastic Model}

For this initial validation we can compare results for each flexibility mode. This allows for the proposed model to be validated against the Wagner results, commonly used in the industry for flexible aircraft development. Wagner results are used as a baseline for numerical comparison, as expected, when there aren't LEV being shed the both Wagner and LDVM match. The results differ when LEV are shed in the flow, which causes the low-order model to converge to an LCO whereas Wagner diverges to infinity. Finally, comparing the low-order model to the Wagner model ultimately helps perceive the non-linear effect of LEV modulation. Some insight on the LESP Critical values can also be obtained from this comparisons, however the definition of the best LESP Critical value for the LDVM model is obtained by verifying against the experimental results.

Looking for the first mode, on figure 9, it is possible to verify that the results from LDVM match those of Wagner when both methods operate on the linear space, i.e. before $3 \mathrm{~s}$. As we reduce the LESP critical, LDVM achieves non-linear territory sooner and presents differences from Wagner. In this 
case it is possible to verify that LESP critical of 0.01 and 0.02 detaches from the standard before the initial transient response is dissipated. LESP critical of 0.01 presents a higher amplitude in the transient response which indicates that leading-edge vortices are being produced from the initial position of one degree of angle of attack. As for the LESP critical of 0.02 the results indicate that LEV shedding starts right after the first transient oscillation.

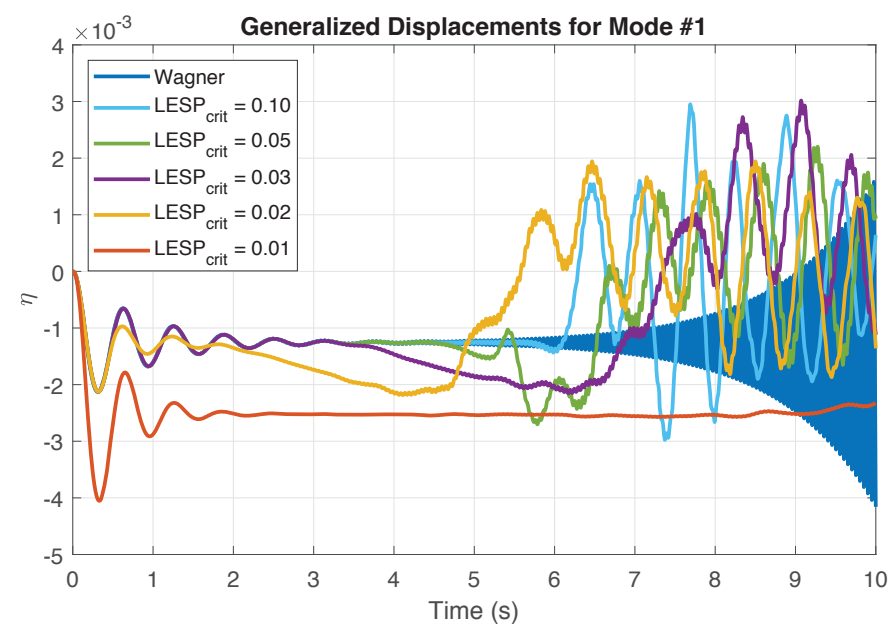

Figure 9: Generalized Displacements - First Mode

Wagner results present divergent behavior that is induced from other modes of flexibility, visible in figures 10 and 11. This explains the higher frequency of the divergent behavior. All LESP values produce movements of limited amplitudes for the first mode of flexibility. And, as is the case for Wagner, it is possible to visualize the influence of the higher frequency modes of flexibility after some seconds of simulation. This coupling between the vibration modes is caused by the aerodynamic model that distributes the energy across all modes. LESP Critical of 0.01 produces a higher amplitude from the start, which indicates that leading-edge vortices are being produced from the initial position of one degree of angle of attack and are always-on. This result is important because it is the modulation of LEV shedding that introduces the non-linear behavior necessary to achieve an LCO, therefore it is possible to get some insight in the minimal LESP Critical values applicable to this model. LESP Critical of 0.02 also detaches from the standard result before the initial transient response finishes, but more results are needed to completely rule out this value.

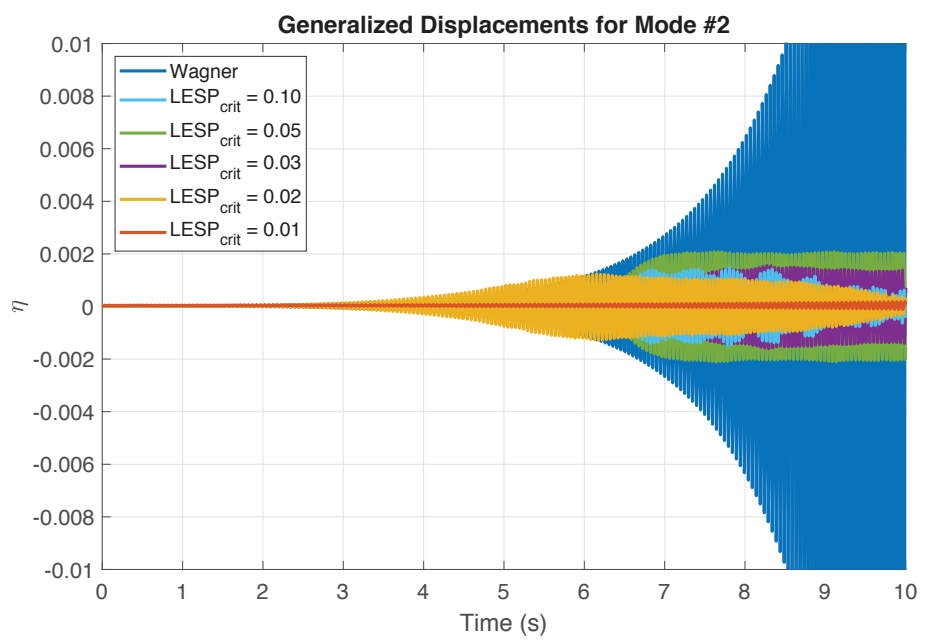

Figure 10: Generalized Displacements - Second Mode

Analyzing now the second flexibility mode (figure 10), it is possible to see that LDVM reaches a 
bounded amplitude for all values of LESP. Wagner, as expected, diverges with increasing values of amplitude for this mode, validating the assumption made before. Also, for the second flexibility mode, the results for LESP Critical equal to 0.10, the baseline value, presents a smaller amplitude than the results from LESP Critical 0.05 and 0.03. The reason for this behavior is due to the influence of the third flexibility mode (figure 11), where the results for critical LESP value of 0.10 diverges to amplitudes much higher than the other. We can also see that LESP Critical of 0.02 eventually converges to an amplitude close to the 0.01 result. This further indicates the assumption made before that the value of 0.02 was indeed too low, as was the case for LESP Critical of 0.01. Finally, the results from the second mode indicate a clear LCO being achieved by the LDVM model for critical LESP values of 0.05 and 0.03.

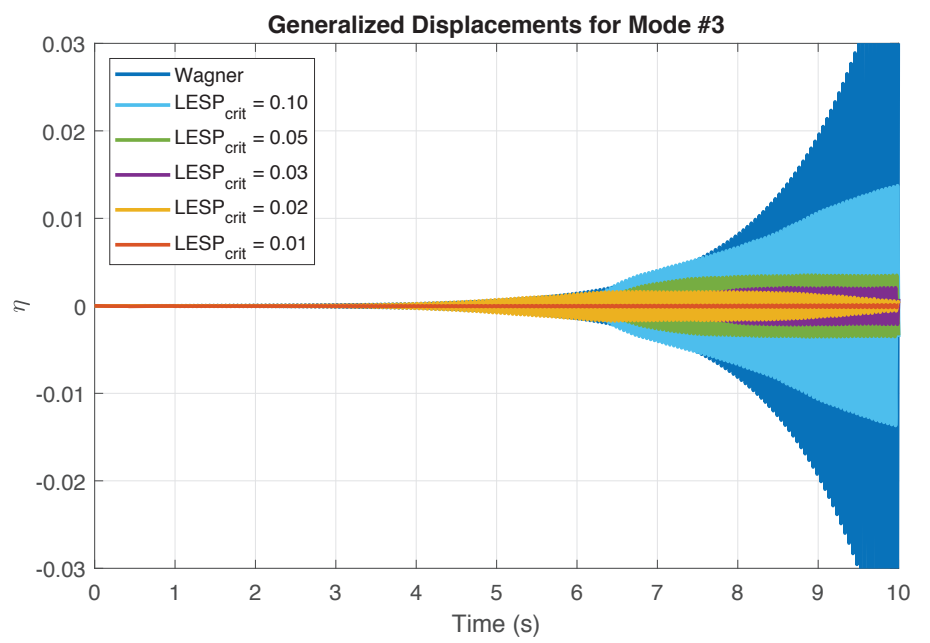

Figure 11: Generalized Displacements - Third Mode

The third flexibility mode results, figure 11, further confirms the possibility of achieving a LCO with the LDVM implementation. It is also possible to verify that the 0.10 value for LESP Critical, which is the baseline value of $2 \mathrm{D}$ applications, does not converge in the simulated time, achieving values that are high enough to interfere with the second mode results. As was the case for the second flexibility mode, in the third flexibility mode, for critical LESP values of 0.01 and 0.02 , the model converges to a stable, non-oscillatory situation. Since the second and third modes form the basis of the flutter LCO that was observed in the experiment, both of these values can be considered too low. This can also be verified by the way they differ from the results of the wagner-based model, which can correctly point the flutter vibration modes, but is unable to converge to the LCO observed in the experiment (figure 17).

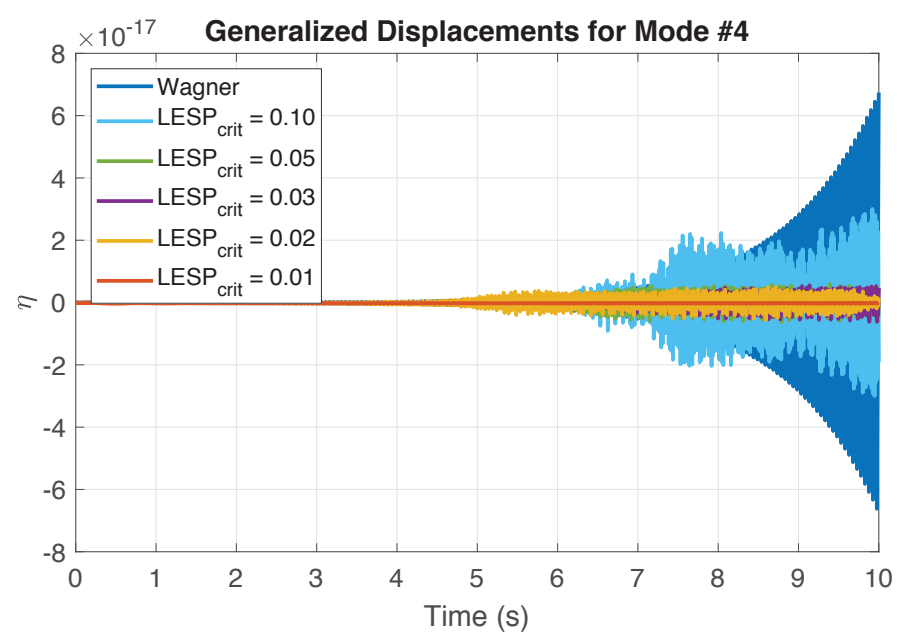

Figure 12: Generalized Displacements - Fourth Mode 
Finally, results for the fourth flexibility mode (figure 12), show that it is not excited by the flutter behavior (the scale of the graph is in 1e-17 order of magnitude). This consolidates the choice of including only 4 flexibility modes in this analysis, since the next mode would not be relevant to the experimental validation. All values are well below the acceptable numerical error, with even the unstable Wagner results not reaching values greater than 1e-16. From a physical perspective this behavior is explained by the increased inertia in the chordwise direction. This would require more energy to produce any vibrations. The linear method divergent behavior ends up achieving higher amplitudes, but the non-linear models keep the energy at a steady level, which produces the LCO, and therefore don't have enough energy to excite this mode.
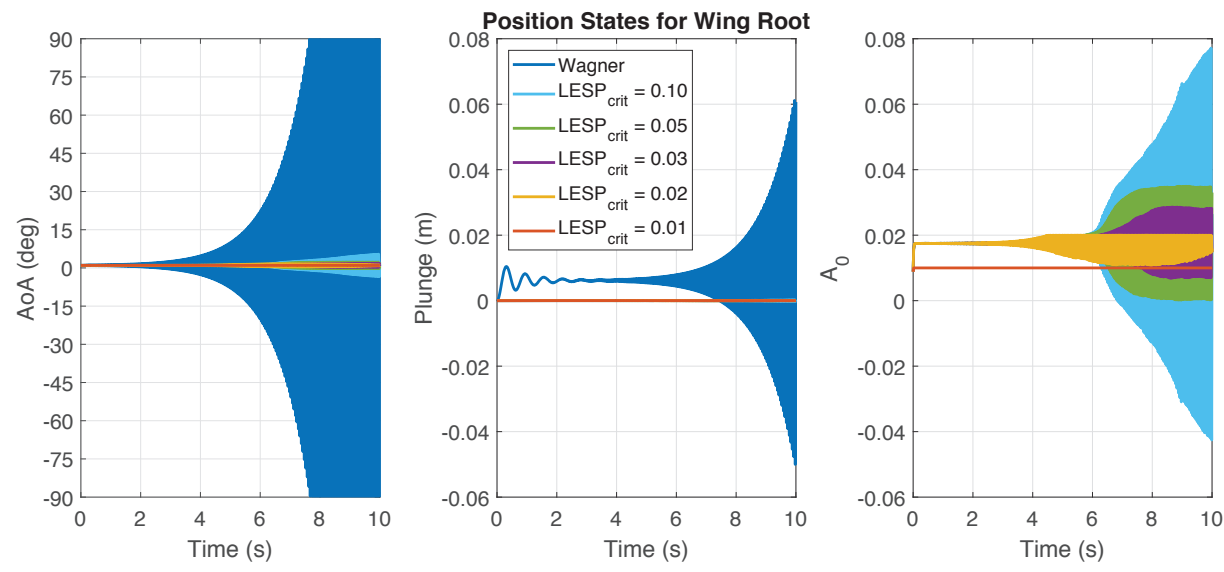

Figure 13: Wing Root Strip States

The actual states of the strips can be obtained from the flexibility modes. For the wing root, measured at the center of the first strip at $0.01 \mathrm{~m}$ of span, figure 13 shows on the right that the $A_{0}$ does not achieve the critical LESP value in most simulations. For instance, the $A_{0}$ converges to value below the corresponding critical LESP values for the 0.10, 0.05 and 0.03 simulations. This is expected, since the fixed wing root have a very restricted movement and should not present high amplitudes in its movement. The graphics at the left and center show that the LESP modulated models keep the angle of attack and plunge at very low values. Figure 13 also confirms the hypothesis that LESP Critical of 0.01 and 0.02 were too low, with the $A_{0}$ value of 0.01 being achieved from the initial condition alone, putting this case in a constant LEV creation routine, and with the $A_{0}$ value of 0.02 being achieved with very low deformations. The behavior visualized for critical LESP value of 0.02 in figure 10 and 11, where modes 2 and 3, respectively, converged out of the LCO after some time, could be linked by LEV generation occurring at all strips, as figure 13 shows. Furthermore, the $A_{0}$ amplitude for the LESP Critical of 0.1 is still increasing, unlike the values for LESP Criticals 0.05 and 0.03 , that converge to fixed amplitudes. This helps verify that the LESP Critical Value of 0.10 is probably too high for the simulated case. As expected, the linear nature of the Jone's approximation makes the Wagner solution diverge even at the wing root.

When analyzing the states for wing tip (figure 14), measured at the center of last strip at $0.39 \mathrm{~m}$ of span, it is clear that LESP Critical of 0.10 is too high for this model, since the last strip exceeds the maximum angles of attack for a feasible system of 90 degrees (figure 14 on the left). This confirms that this value is indeed not able to achieve a feasible LCO. This is important because it means that by simply analyzing the results of the model against the standard linear solution, it is possible to perceive minimum and maximum values for the critical LESP parameter. The results from LESP Critical 0.05 and 0.03 are within the feasible design space with angles between -90 and 90 degrees. From right graphic of figure 14 we can also see that they modulate correctly between the critical values, which indicates the shedding of leading-edge vortices is correctly controlled by the proposed model.

The vortex map (figure 15) provides a general picture of the overall aerodynamic flow, and the flexibility influence in the wake and LEV generation. The figures were generated for the LESP Critical of 0.02 in three different moments of the simulation. Strips located near the wing tip (higher values of y) present higher motion amplitude with the wake showing a clearer oscillatory trajectory than strips closer to the wing root. It is also possible to visualize, from the three time snapshots, gaps in the LEV distribution in the $\mathrm{X}$ axis due to the modulation of LEV generation. And Finally, for the LESP Critical of 0.02 , leading edge vortices are generated in all strips, although that can happen in different moments 

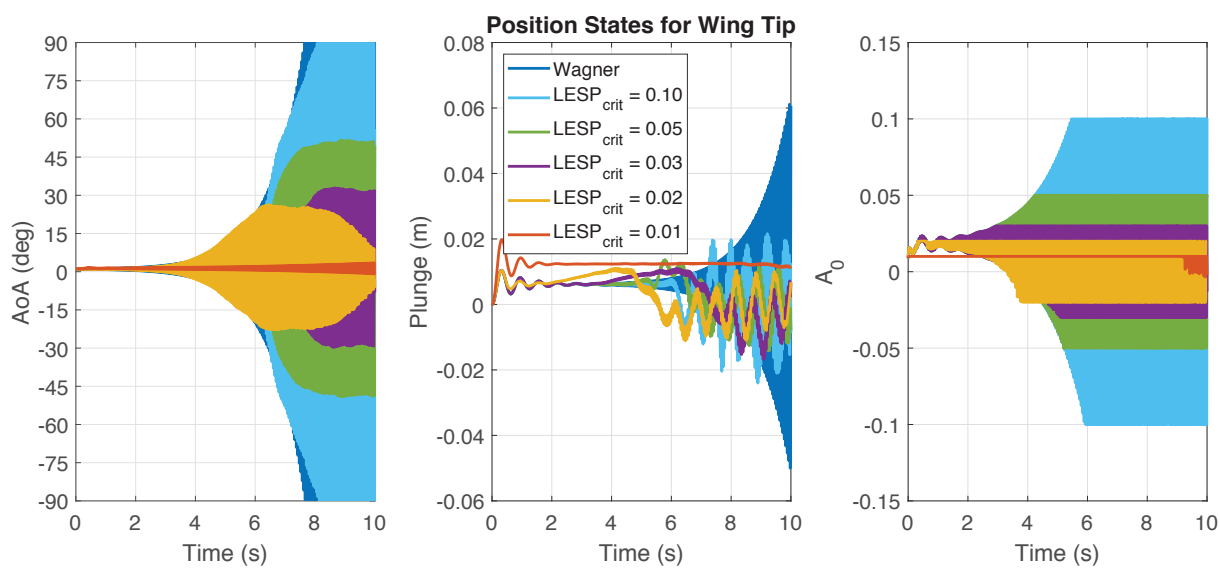

Figure 14: Wing Tip Strip States
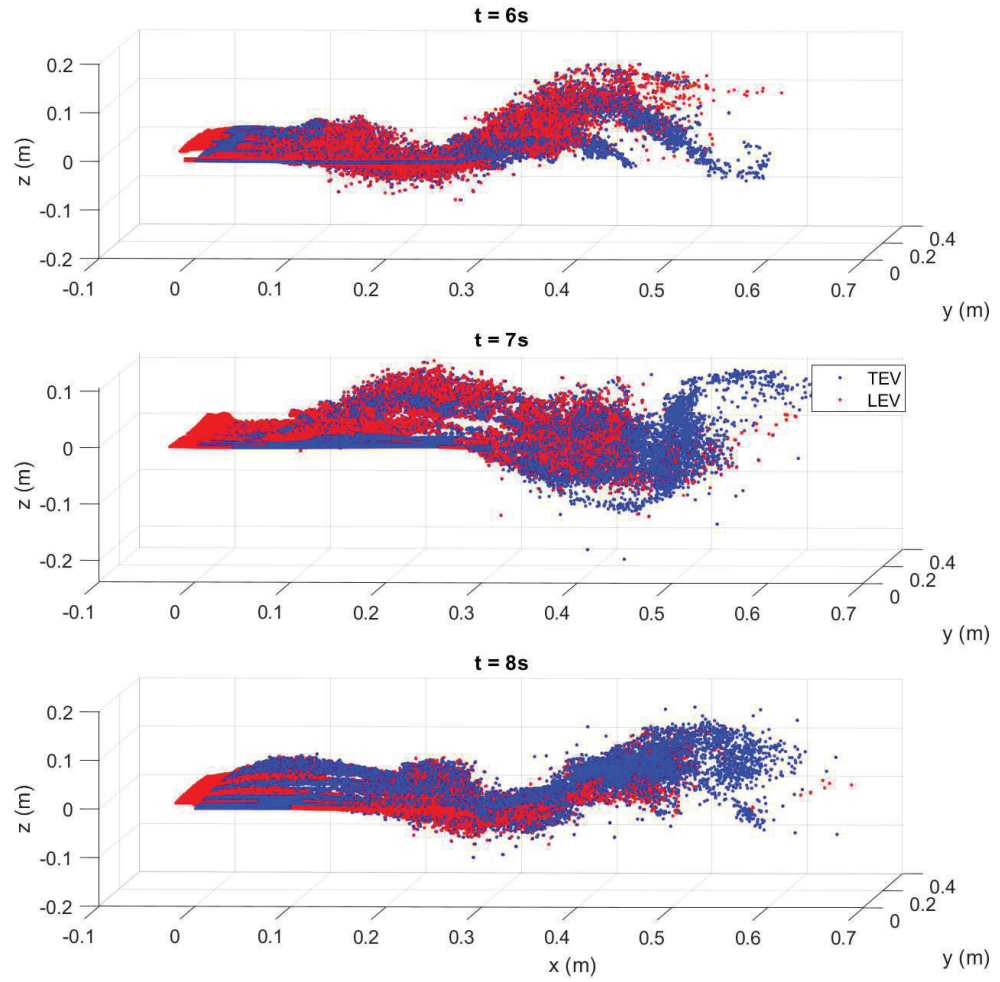

Figure 15: Wing Vortex Map 
in time. Looking closely at the strips at the root, middle and tip of the wing for the 6s (figure 16) it is possible to visualize the differences in the wake caused by the elastic behavior of the wing, where: The wing root presents a very well behaved wake comprised of LEVs and TEVs, which is expected due to the lack of movement and the aforementioned use of low LESP critical value. The middle of the wing presents a positive plunge displacement coupled with a negative pitch, the amplitude of the motion, evidenced by the vortex distribution on the wake when compared to the tip strip. Finally, the wing tip show the highest amplitude of motion and, at the $6 \mathrm{~s}$ mark, presents a negative plunge and pitch scenario. The wing picture to the right of figure 16 shows mainly the presence of the second and third flexibility modes due to the curvature at the middle and the pronounced angle of attack near the tip.
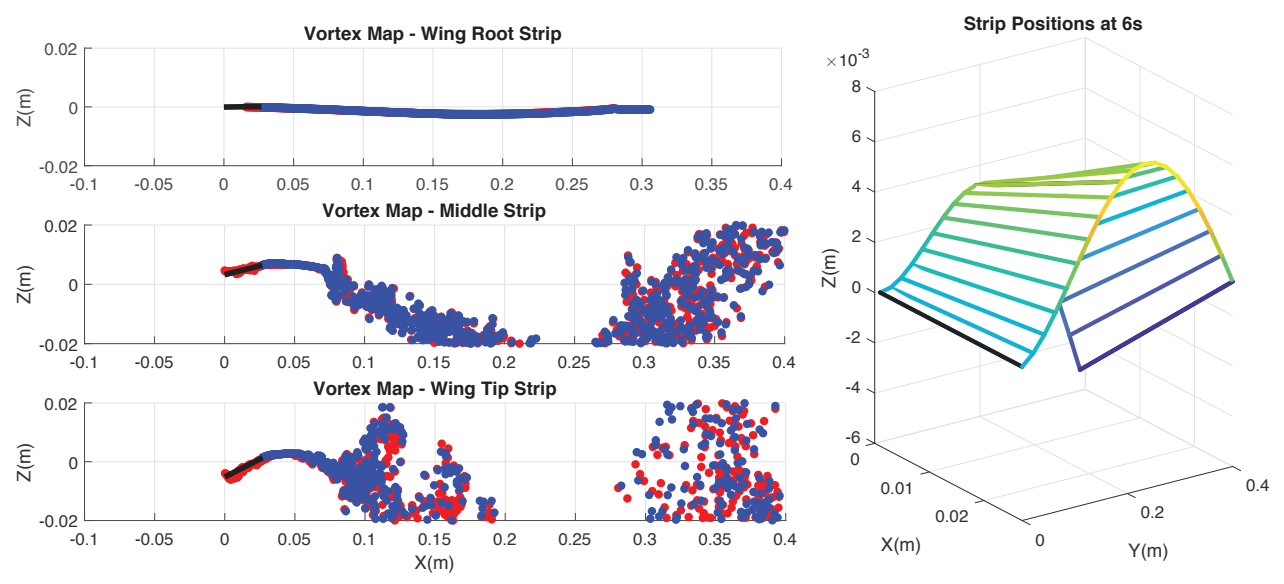

Figure 16: Wing Vortex Map - Zoom

\subsection{Comparison with Experimental results}

For this validation the velocity measured at the trailing edge position was used.

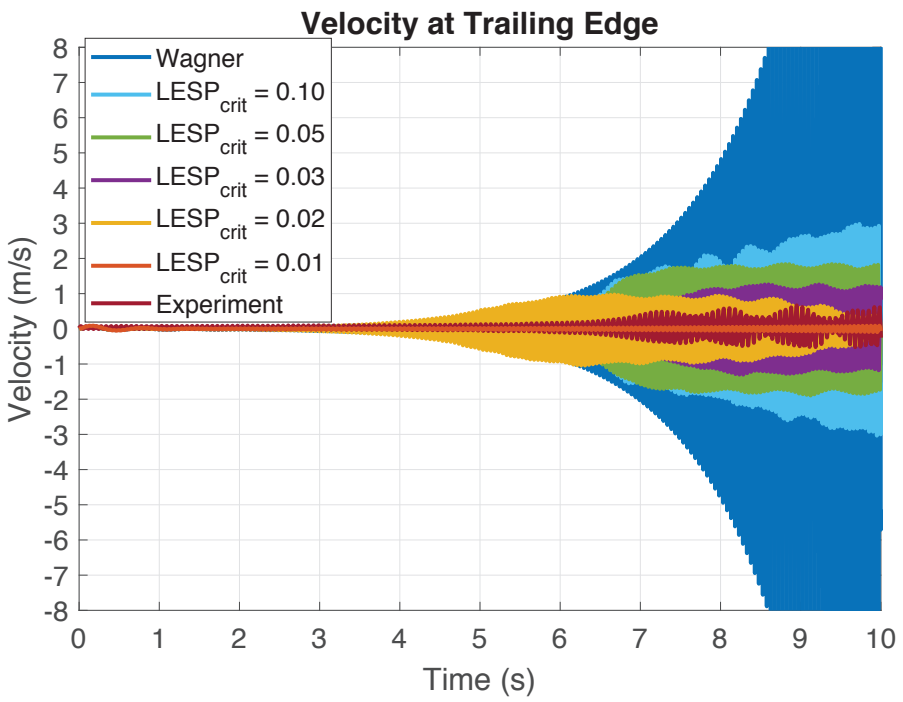

Figure 17: Trailing Edge Velocity

Figure 17 reveals that, for the velocity of $10 \mathrm{~m} / \mathrm{s}$, defined in the experiment as the flutter velocity, the results for LDVM present a higher amplitude for both cases where an LCO is achieved (LESP Critical of 0.03 and 0.05). As expected, LESP Critical of 0.01 does not achieve an LCO, due to the always-on nature of the leading-edge vortex shedding. And LESP Critical of 0.02, eventually converges out of the 
LCO due to all its strips having leading-edge vortex shedding. For the LESP critical of 0.03 and 0.05 , the model captures the general trends the LCO behavior and influence between the first mode and the second and third modes LCO. This a major result, not possible with other linear methods. From these results LESP Critical of 0.03 should be considered the correct model setup, since it presents a better fit for the amplitudes found by the experiment than the LESP critical value of 0.05 . The differences in amplitude are however expected from the limitations described for both, the model and experiment. Simulating further in time the results for LESP Critical 0.02 and 0.03 (figure 18), it is possible to see that the results for LESP Critical 0.02 are over damped and end up filtering out the higher frequencies, whereas the LESP Critical 0.03 converges to a amplitude very close to the experimental result.

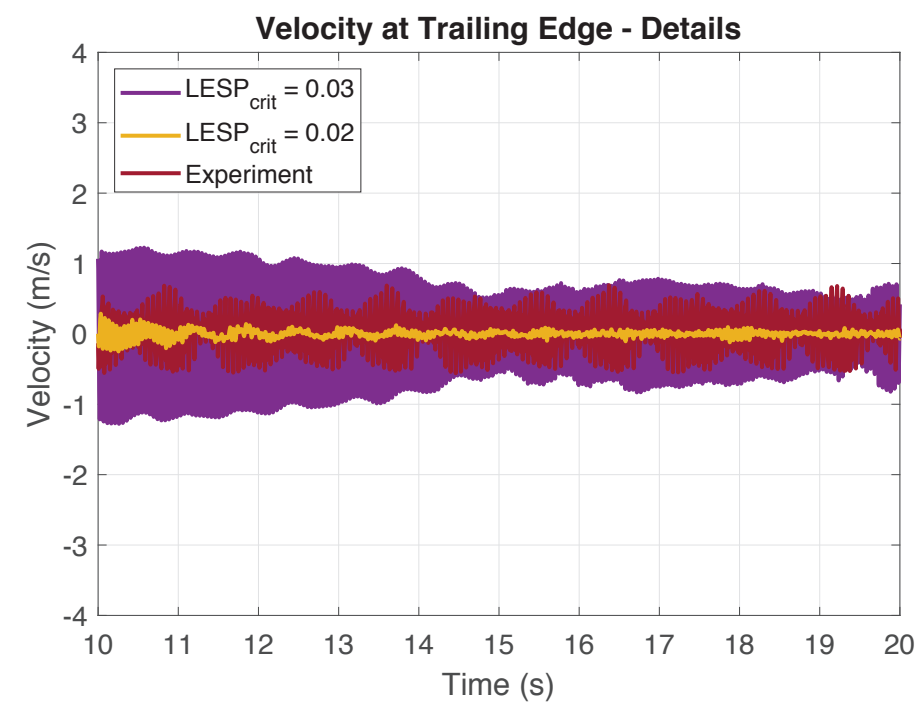

Figure 18: Trailing Edge Velocity - Extended Results
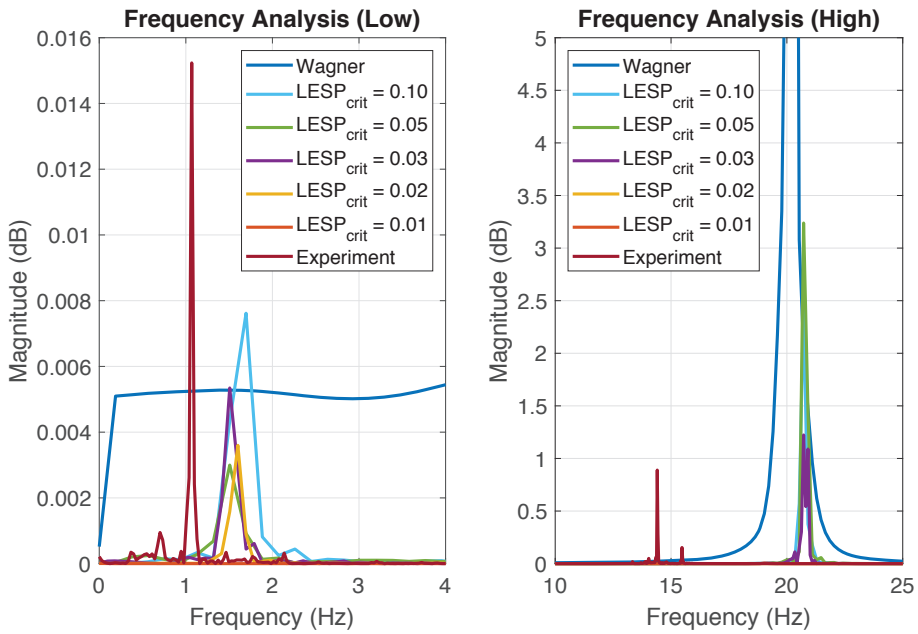

Figure 19: Trailing Edge Velocity - Frequency Spectrum

When looking at the frequency analysis it is possible to see a variation between the model frequencies and the ones measured at the experiment (figure 19). In the left-side graphic, the experiment presents a low frequency peak at around $1 \mathrm{~Hz}$, whereas the non-linear models concentrate the low frequency peak around $1.5 \mathrm{~Hz}$. This difference can be attributed to the limitations of the modal approximation and aerodynamics model, and are acceptable for a low-order model, specially when compared to the linear, which can't reproduce this low frequency result at all. The amplitude of the energy is also different between the model and experiment, which is probably due to the lack of $3 \mathrm{D}$ effect at the wing tip 
and some influence of aerodynamic balance vibration. In the right-side graphic, we also see the models reaching a higher frequency than the experiment, with the former presenting a peak around $14.5 \mathrm{~Hz}$ and the latter presenting a peak around $20.5 \mathrm{~Hz}$. This difference is explained by the influence of the aerodynamic balance, that is not modeled by the aeroelastic model. As observed in the complete apparatus FEM model, the balance has the effect of introducing an additional vibration mode at $14 \mathrm{~Hz}$, which plays a major role in the flutter's LCO high frequency component. The wing modes, which are tightly coupled with this additional mode present a smaller peak dislocated to around $15.5 \mathrm{~Hz}$. As verified by the timebased analysis the LESP Critical of 0.03 presents the best fit for the high frequency amplitude. The linear method shows a single peak, of a much higher amplitude, due to its divergent behavior. Finally, figure 20 focus on the latter part of the time response (17-19s) to help visualize the differences between experiment and the 0.03 LESP Critical results. It is possible to see the influence of the higher amplitude in the low-frequency response of the experiment, with the highs and lows more pronounced than the simulation results.

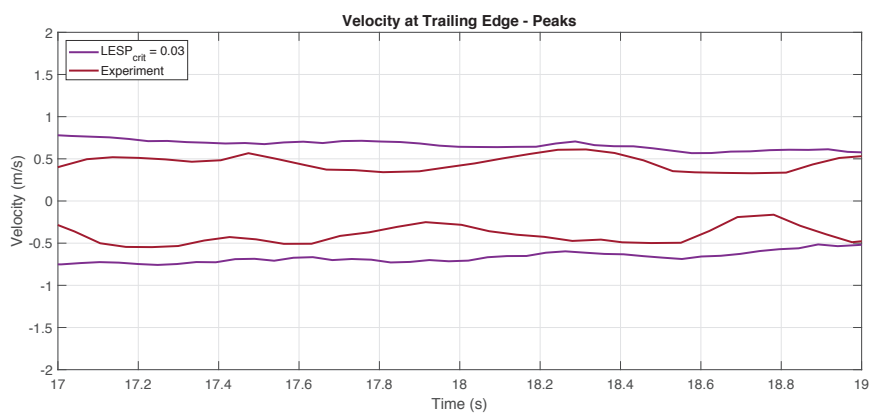

Figure 20: Trailing Edge Velocity - Peaks Plot

Finally, a phase plane analysis (figure 21) was developed based on the measured velocity from the laser vibrometer. Since, a displacement signal was not captured from this device, the velocity signal between $17 \mathrm{~s}$ and $19 \mathrm{~s}$ was integrated over time to generated this result. Figure 21 further confirms that the proposed model is able to capture the general tendencies of the LCO, with the oscillation of the displacement around the zero position being due the influence of the first mode of vibration, stronger on the experiment than on the model as seen on the frequency response, and the aerodynamic balance influence that was discussed before. The wind tunnel turbulence level can also play a role in the differences seen in the displacement range. Also, as seen on the time-based results, there is an overestimation of the velocity by the model likely due to the lack of $3 \mathrm{D}$ effects in the model discussed before.

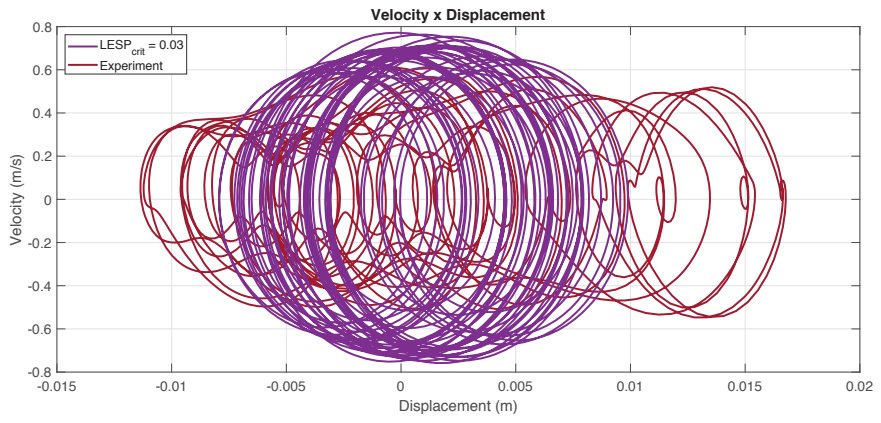

Figure 21: Phase Plane

The lifting line correction for 3D applications, studied by Ramesh et al. [33] previously, can affect this results and help understand the nature of this differences, specifically the amplitude differences. Further studies with Unsteady Vortex Lattice Methods, augmented with the LESP concept, are in development and should help understand the 3D effects influence in this results as well. 


\section{Conclusions}

The proposed framework of combining modal superposition with a strip theory implementation LDVM was able to achieve a sustainable LCO and capture behaviors that can be compared to the experiment's measured amplitudes, including the modal interaction between the flutter flexibility modes (modes 2 and 3 ) and the first bending mode (mode 1). This solves a major gap in flexible aircraft modeling where engineers need to use costly CFD models to represent the aerodynamic non-linearities encountered by these aircraft. Time-based and frequency-based results show that the models can approximate low and high frequency LCO response with a relative low-cost model. The main differences observed between the experimental results and the low-order model are explained by two of the model limitations, i.e. the aerodynamic balance, that is not modeled by the structural model, explains the differences in frequency and also affects the low-frequency amplitude, whereas the lack of 3D effects, specially wingtip vortex effect, also affects the amplitude, specially for the first mode response.

The proposed framework can be executed in desktop PCs with relative low computation cost, taking about 2 days to develop the 10s simulations for the stable cases of LESP Critical 0.03 and 0.05. Comparisons with linear models, such the standard Wagner formulation, can be used to help tune, although are not sufficient, the non-linear behavior of the LDVM model for 3D applications at a fraction of the time that would be required for full CFD/FEM simulations. Also, the move from the 2D airfoil implementation to the strip theory model shows that changes to the critical LESP need to be made to achieve convergence to a LCO. Finally, the model proposed also presents a scalable nature to more complex problems through the use of parallel computing for each individual strip.

Results from the proposed model are directly comparable to the standard Wagner approximation method used for the linear results, diverging and reaching the LCO when the aerodynamic non-linearities begin to act. This means that a simplified version of the framework (with very high LESP Criticals) can be used to assess the probability of a aerodynamic linear solution being unable to correctly represent situations where leading-edge vortices might be present.

Future works are planned with lifting line implementation and UVLM, to help mitigate some of the amplitude differences between the model proposed and the experimental data in the aerodynamic side, whereas an expanded nonlinear structural formulation should make the model more robust for the cases of large structural deformations.

\section{Acknowledgment}

This study was financed in part by the Coordenação de Aperfeiçoamento de Pessoal de Nível Superior Brasil (CAPES) - Finance Code 001

\section{References}

[1] Silvestre, F. J., Guimarães Neto, A. B., Bertolin, R. M., et al., Aircraft control based on flexible aircraft dynamics., Journal of Aircraft, 2016.

[2] Murua, J., Palacios, R., Graham, J. M. R., Applications of the unsteady vortex-lattice method in aircraft aeroelasticity and flight dynamics, Progress in Aerospace Sciences, 2012

[3] Hesse, H., Palacios, R., Reduced-Order Aeroelastic Models for Dynamics of Maneuvering Flexible Aircraft, AIAA Journal, 2014

[4] Silvestre, F. J., Luckner, R., Experimental Validation of a Flight Simulation Model for Slightly Flexible Aircraft, AIAA Journal, 2015

[5] Palacios, R., Murua, J., Cook, R., Structural and Aerodynamic Models in Nonlinear Flight Dynamics of Very Flexible Aircraft, AIAA Journal, 2010.

[6] Theodorsen, T., General theory of aerodynamic instability and the mechanism of flutter., NACA Rept. 496, 1935.

[7] Theodorsen, T. and Garrick, I. E., Flutter calculations in three degrees of freedom., NACA Rept. $741,1942$. 
[8] Wagner, H., Über die Entstehung des dynamischen Auftriebes von Tragflügeln, ZaMM, 5(1), 17-35, 1925.

[9] Denegri, C. M., Dubben, J. A., and Maxwell, D. L., In-flight wing deformation characteristics during limit cycle oscillations., Journal of Aircraft, 42(2), 500-508, 2005.

[10] Leishman, J. G. and Beddoes, T. S., A semi-empirical model for dynamic stall., Journal of the American Helicopter Society, 34(3), 3-17, 1989.

[11] Petot, D., Progress in the semi-empirical prediction of the aerodynamic forces due to large amplitude oscillations of an airfoil in attached or separated flow., Tech. Rep. 1983-111, 1983.

[12] Shearer, C. M., Cesnik, C.E.S., Nonlinear Flight Dynamics of Very Flexible Aircraft, Journal of Aircraft, 2007.

[13] Ramesh, K., Gopalarathnam, A., Granlund, K., et al., Discrete-vortex method with novel shedding criterion for unsteady airfoil flows with intermittent leading-edge vortex shedding., Journal of Fluid Mechanics, 751, 500-538, 2014.

[14] Wang, C. and Eldredge, J. D., Low-order phenomenological modeling of leading-edge vortex formation., Theoretical and Computational Fluid Dynamics, 27(5), 577-598, 2012.

[15] Lee, B. H. K., Price, S. J., and Wong, Y. S., Nonlinear aeroelastic analysis of airfoils: bifurcation and chaos., Progress in Aerospace Sciences, 35(3), 205-334, 1999.

[16] Tang, D. M., Yamamoto, H., and Dowell, E. H., Flutter and limit cycle oscillations of twodimensional panels in three-dimensional axial flow., Journal of Fluids and Structures, 17(2), 225-242, 2003.

[17] Leishman, J. G., Principles of Helicopter Aerodynamics, Cambridge Aerospace Series, 2002.

[18] Ellington, C. P., Unsteady aerodynamics of insect flight., Symposia of the Society for Experimental Biology, vol. 49. p.109, 1995.

[19] Shyy, W. and Liu, H., Flapping wings and aerodynamic lift: The role of leading-edge vortices., AIAA Journal, 45(12), 2007.

[20] Ellington, C. P., The novel aerodynamics of insect flight: applications to micro-air vehicles., Journal of Experimental Biology, 202(23), 3439-3448, 1999.

[21] Dickinson, M. H. and Gotz, K. G., Unsteady aerodynamic performance of model wings at low Reynolds numbers, Journal of Experimental Biology, 174(1), 45-64, 1993.

[22] Anderson, J., Streitlien, K., Barrett, D., et al., Oscillating Foils of High Propulsive Efficiency, Journal of Fluid Mechanics, 360(1), 41-72, 1998.

[23] Kinsey, T. and Dumas, G., Parametric study of an oscillating airfoil in a power-extraction regime., AIAA Journal, 46(6), 1318-1330, 2008.

[24] Dowell, E. H. and Tang, D., Nonlinear aeroelasticity and unsteady aerodynamics., AIAA journal, 40(9), 1697-1707, 2002.

[25] Ramesh, K., Murua, J., and Gopalarathnam, A. Limit-cycle oscillations in unsteady flows dominated by intermittent leading-edge vortex shedding., Journal of Fluids and Structures, 55, 84-105, 2015.

[26] Tang, D., Dowell, E. H., and Hall, K. C., Limit cycle oscillations of a cantilevered wing in low subsonic flow.,AIAA journal, 37(3), 364-371, 1999.

[27] Preidikman, S., Mook, D., Time-Domain Simulations of Linear and Nonlinear Aeroelastic Behavior, Journal of Vibration and Control, 2000.

[28] Patil, M. J., Hodges, D. H., Cesnik, C. E. S., Nonlinear Aeroelasticity and Flight Dynamics of High-Altitude Long-Endurance Aircraft, Journal of Aircraft, 2001.

[29] Ghadiri, B. and Razi, M., Limit cycle oscillations of rectangular cantilever wings containing cubic nonlinearity in an incompressible flow., Journal of Fluids and Structures, 23(4), 665-680, 2007. 
[30] Tang, L., Païdoussis, M. P., and Jiang, J. Cantilevered flexible plates in axial flow: energy transfer and the concept of flutter-mill., Journal of Sound and Vibration, 326(1), 263-276, 2009.

[31] Ramesh, K., Gopalarathnam, A., Edwards, J. R., et al., An unsteady airfoil theory applied to pitching motions validated against experiment and computation., Theoretical and Computational Fluid Dynamics, 27(6), 843-864, 2013.

[32] Ramesh, K., Gopalarathnam, A., Ol, M. V., et al., Augmentation of inviscid airfoil theory to predict and model 2D unsteady vortex dominated flows, AIAA Paper, 2011-3578, 2011.

[33] Ramesh, K., Monteiro, T. P., Silvestre, F., et al., Experimental and numerical investigation of postflutter limit cycle oscillations on a cantilevered flat plate, IFASD, 2017.

[34] Hirato, Y., Leading-Edge-Vortex Formation on Finite Wings in Unsteady Flow, North Carolina State University, 2016.

[35] Monteiro, T. P., Ramesh, K., Silvestre, F., Numerical Development of Unsteady Vortex-Lattice Method Expansion for Leading-Edge Shedding Prediction with Experimental Validation, ICAS, 2018.

[36] Katz, J., Plotkin, A., Low-Speed Aerodynamics, Cambridge Aerospace Series, 2000.

[37] Garrick, I. E., Propulsion of a flapping and oscillating airfoil., NACA Rept. 567, 1937.

[38] von Kármán, T., Burgers, J. M., General Aerodynamic Theory - Perfect Fluids, vol. 2 of Aerodynamic theory: a general review of progress, Dover Publications, 1963.

[39] Katz, J., Discrete vortex method for the non-steady separated flow over an airfoil., Journal of Fluid Mechanics, 102(1), 315-328, 1981.

[40] Vatistas, G. H., Kozel, V., and Mih, W. C., A simpler model for concentrated vortices., Experiments in Fluids, 11(1), 73-76, 1991.

[41] SureshBabu, A., Ramesh, K., Gopalarathnam, A., Model Reduction in Discrete-Vortex Methods for Unsteady Airfoil Flows, AIAA Journal, 2019.

[42] Darakananda, D., Silva, A.F.C., Colonius, T., Eldredge, J.D., Data-assimilated low-order vortex modeling of separated flows, Physical Review Fluids, 2018.

[43] Chandrupatla, T., Belegundu, A., Introduction to Finite Elements in Engineering, Prentice Hall, 2002.

[44] Schmidt, D. K., Modern Flight Dynamics, McGraw-Hill, New York, 2012, pp. 128-155.

[45] Bisplinghoff, L. R., Ashley, H., Halfman, L. R., Aeroelasticity, Dover Publications, 1996.

[46] Eldredge, J. D., Jones, A. R., Leading-Edge Vortices: Mechanics and Modeling, Annual Review of Fluid Mechanics, 2019. 